\title{
Monte Carlo construction of cubature on Wiener space
}

\author{
Satoshi Hayakawa ${ }^{1}$ (D) Ken'ichiro Tanaka
}

Received: 10 March 2021 / Revised: 8 November 2021 / Accepted: 22 November 2021 /

Published online: 27 December 2021

(c) The Author(s) 2021

\begin{abstract}
In this paper, we investigate application of mathematical optimization to construction of a cubature formula on Wiener space, which is a weak approximation method of stochastic differential equations introduced by Lyons and Victoir (Proc R Soc Lond A 460:169-198, 2004). After giving a brief review on the cubature theory on Wiener space, we show that a cubature formula of general dimension and degree can be obtained through a Monte Carlo sampling and linear programming. This paper also includes an extension of stochastic Tchakaloff's theorem, which technically yields the proof of our primary result.
\end{abstract}

Keywords Weak approximation of SDE - Cubature on Wiener space - Lie algebra · Tchakaloff's theorem · Monte Carlo sampling

\section{Introduction}

Cubature on Wiener space [19] is a certain family of numerical formula for approximating the expectation of functionals of diffusion processes, which are important in mathematical finance and other related fields. The existence of cubature formula of general dimension and degree has been known, but the constructions given in the literature were based on algebraic structure among continuous paths and Brownian motion, and limited to ones of low degree and dimension. Our aim in this paper is to obtain a method of constructing general cubature formula on Wiener space through mathematical optimization.

More concretely, we are interested in approximating values in the form $\mathrm{E}\left[f\left(X_{T}\right)\right]$, where $\left(X_{t}\right)_{0 \leq t \leq T}$ is a solution of stochastic differential equation (SDE) driven by a

Satoshi Hayakawa

hayakawa@maths.ox.ac.uk

1 Mathematical Institute, University of Oxford, Andrew Wiles Building, Radcliffe Observatory Quarter, Woodstock Road, Oxford OX2 6GG, UK

2 Graduate School of Information Science and Technology, The University of Tokyo, 7-3-1, Hongo, Bunkyo-ku, Tokyo, Japan 
multidimensional Brownian motion, and $f$ is a function with some regularity (e.g., Lipschitz continuity). The standard approach consists of two steps:

(1) approximate the distribution of $X_{t+\Delta t}$ from the information of (approximated) $X_{t}$, where $\Delta t \ll 1$;

(2) split the time interval $[0, T]$ by $0=t_{0}<t_{1}<\cdots<t_{k}=T$ and sequentially apply (1) over each $\left[t_{\ell}, t_{\ell+1}\right]$.

For example, the stochastic process $\tilde{X}_{T}^{\mathrm{EM},(k)}$ given by the Euler-Maruyama method with equal partition attains $\left|\mathrm{E}\left[f\left(\tilde{X}_{T}^{\mathrm{EM},(k)}\right)\right]-\mathrm{E}\left[f\left(X_{T}\right)\right]\right|=\mathrm{O}(1 / k)$ with respect to $k$, the number of partitions [12].

To achieve higher precision of approximation such as $\mathrm{O}\left(1 / k^{2}\right)$, Lyons and Victoir [19] introduced the cubature on Wiener space, which uses the higher order of stochastic Taylor expansion in step (1). The basic scheme for (1) in cubature on Wiener space is as follows:

(c1) approximate the distribution of Brownian motion $\left(B_{s}\right)_{t \leq s \leq t+\Delta t}$ by a weighted discrete set of deterministic paths;

(c2) locally (over $[t, t+\Delta t]$ ) solve ordinary differential equations (ODEs) driven by the deterministic paths in (c1) instead of the stochastic differential equations for approximating the distribution of $X_{t+\Delta t}$;

Among the steps (c1), (c2), (2) in cubature on Wiener space, this paper primarily focuses on finding a good weighted set of deterministic paths described in (c1).

In the field of high-order approximation of SDEs, there is also a relative of cubature on Wiener space called Kusuoka approximation [15] using a random paths in (c1), which is followed by the papers [20, 22] presenting concrete second-order schemes. The main challenge shared by these approaches (cubature on Wiener space, Kusuoka approximation) is that constructing such a (random) set of paths has been performed by actually solving Lie-algebraic equations and is limited to low dimension and degree of precision. Therefore, our objective is to find a way of generally constructing such a formula in arbitrary dimension and degree.

Contribution of this study Broadly speaking, the contribution of this study comprises the following two items:

- (main contribution) We show that one can construct a cubature formula on Wiener space of general dimension and degree with a randomized algorithm.

- (technical contribution) To apply the technique of [10] to the problem of cubature on Wiener space, we characterize the affine hull of the distribution of iterated Stratonovich integrals and prove stochastic Tchakaloff's theorem in a stronger way.

The main result with a simple Monte Carlo construction is given in Proposition 19. It asserts that a certain random generation of piecewise linear paths combined with a linear programming yields a cubature formula on Wiener space. 
As a technical contribution, we extend stochastic Tchakaloff's theorem [19], which assures the existence of cubature formulas on Wiener space. Although the original statement was just that there exists a cubature formula, we show that the expectation of iterated Stratonovich integrals of a Brownian motion $\left(\mathrm{E}\left[\boldsymbol{\varphi}_{\mathrm{W}}(B)\right]\right.$ in (4)) is contained in the relative interior of $\operatorname{conv}\left\{\boldsymbol{\varphi}_{\mathrm{W}}(w)\right\}$ with a valid range of bounded variation (BV) paths $w$ (Theorem 17). This stronger statement with "relative interior" follows our characterization (Proposition 15) of aff $\operatorname{supp} \mathrm{P}_{\varphi_{\mathrm{W}}(B)}$ in terms of (4) and is essential in exploiting the existing construction of general cubature formula [10].

We only treat the part (c1) in this study, but it is important to consider combining our construction with ODE solvers (c2) and some techniques reducing computational complexity (2) such as recombination [17], which needs further investigation and is deferred to future research.

Outline We give a brief overview of the following sections.

Section 2 describes the idea and background of this study in a more mathematical way. We briefly explain the concept of cubature on Wiener space in Sect. 2.1 and the overview of a preceding result recently given by one of the authors [10] in Sect. 2.2.

Section 3 is devoted to a theoretical review of cubature on Wiener space by [19]. We introduce the facts around vector fields and relevant notations in Sect. 3.1 used throughout the paper. The precise definition and error estimate of cubature on Wiener space is given in Sect. 3.2, and Sect. 3.3 provides information about known constructions based on algebraic arguments.

In Sect. 4, we give the extended statement of stochastic Tchakaloff's theorem and its proof. Sections 4.1 and 4.2 provide algebraic background of cubature construction. Section 4.3 is devoted to the proof of our version of stochastic Tchakaloff's theorem, and it also includes the characterization of the distribution of iterated Stratonovich integrals from the viewpoint of the affine hull of the support.

We discuss a way to obtain piecewise linear cubature formula on Wiener space in Sect. 5. After giving some general properties of continuous BV paths in our context in Sect. 5.1, we prove our main result in Sect. 5.2 that we can generally construct cubature formulas on Wiener space with simple randomized algorithms. Section 5.3 represents the numerical verification of our result in a small range of parameters.

Finally we summarize our conclusion in Sect. 6.

\section{Preliminaries}

In this section, we shall briefly explain the background and motivation of this study. Section 2.1 gives a brief explanation of the cubature theory on Wiener space [19] and identifies the problem to be solved. Section 2.2 introduces the recent Monte Carlo approach by [10] to general cubature construction, which our method in this 
paper is based on. We explain the relation between cubature on Wiener space and a generalized one, as well as the particular difficulty that arise on the Wiener space.

\subsection{Cubature on Wiener space}

Let $B=\left(B^{1}, \ldots, B^{d}\right)$ be a $d$-dimensional standard Brownian motion. Let $C_{b}^{\infty}\left(\mathbb{R}^{N} ; \mathbb{R}^{N}\right)$ be the space of infinitely differentiable $\mathbb{R}^{N}$-valued functions defined on $\mathbb{R}^{N}$ whose every order of derivative is bounded. Let us consider the following $N$-dimensional Stratonovich SDE:

$$
\mathrm{d} X_{t}=\sum_{i=1}^{d} V_{i}\left(X_{t}\right) \circ \mathrm{d} B_{t}^{i}+V_{0}\left(X_{t}\right) \mathrm{d} t, \quad X_{0}=x,
$$

where $x \in \mathbb{R}^{N}, V_{i} \in C_{b}^{\infty}\left(\mathbb{R}^{N} ; \mathbb{R}^{N}\right)$ for $i=0, \ldots, d$. As the process $X_{t}$ is dependent on the initial value $x$, we denote it by $X_{t}(x)$ if necessary. We may assume the solution $X_{t}(x)$ is continuous with respect to $t$ and $x$. Our aim is to efficiently compute or approximate the expectation $\mathrm{E}\left[f\left(X_{t}\right)\right]$ with $t>0$ and some smooth or Lipschitz $f$. This sort of approximation is called a weak approximation of SDE and well-studied in the literature $[12,13,15,19,20,22,23]$.

We here focus on the approach introduced in [19] called cubature on Wiener space. Broadly speaking, a cubature formula on Wiener space (of the time interval $[0, T])$ is the approximation

$$
\mathrm{P} \simeq \sum_{i=1}^{n} \lambda_{j} \delta_{w_{j}}
$$

where $\mathrm{P}$ is the Wiener measure on the Wiener space $C_{0}^{0}\left([0, T] ; \mathbb{R}^{d}\right)$ (the space of $\mathbb{R}^{d}$ -valued continuous function in $[0, T]$ starting at the origin),

$$
w_{j}=\left(w_{j}^{0}, w_{j}^{1}, \ldots, w_{j}^{d}\right) \in C_{0}^{0}\left([0, T] ; \mathbb{R} \oplus \mathbb{R}^{d}\right)
$$

for $j=1, \ldots, n$, and $\lambda_{1}, \ldots, \lambda_{n}$ are positive real weights whose sum equals one. Instead of polynomials in conventional cubature formulas, we adopt iterated integrals as the test functionals, that is, we want to find paths $w_{i}$ satisfying

$$
\mathrm{E}\left[\int_{0<t_{1}<\cdots<t_{k}<T} \circ \mathrm{d} B_{t_{1}}^{i_{1}} \cdots \circ \mathrm{d} B_{t_{k}}^{i_{k}}\right]=\sum_{j=1}^{n} \lambda_{j} \int_{0<t_{1}<\cdots<t_{k}<T} \mathrm{~d} w_{j}^{i_{1}}\left(t_{1}\right) \cdots \mathrm{d} w_{j}^{i_{k}}\left(t_{k}\right)
$$

over some set of multiindices $\left(i_{1}, \ldots, i_{k}\right)$, where the iterated Stratonovich integral appears in the left-hand side. Precisely speaking, we formally set $B_{t}^{0}=t$ for $t \geq 0$ and assume $w_{j}$ is a path of $\mathrm{BV}$ for each $j=1, \ldots, n$. Although $w_{j}^{0}(t)=t$ is also assumed in [19], here we may generalize and remove this condition.

The iterated integrals appearing in (2) have a rich algebraic structure (see Sect. 4.1), so algebraic approaches have been adopted in the literature [8, 19, 21 , $22,24,29]$. However, solving complicated equations of Lie algebra is required in those approaches, and constructions of the formula are limited to a small range 
Table $1(d, m)=(2,3)$, $|\mathscr{A}(m)|=20$

\begin{tabular}{llllll}
\hline$N \backslash M$ & 2 & 4 & 8 & 16 & 32 \\
\hline $2|\mathscr{A}(m)|$ & 3 & 4 & 3 & 2 & 2 \\
$4|\mathscr{A}(m)|$ & 10 & 10 & 10 & 10 & 10 \\
$8|\mathscr{A}(m)|$ & & & & & \\
\hline
\end{tabular}

Table $2(d, m)=(3,3)$, $|\mathscr{A}(m)|=47$

\begin{tabular}{llllll}
\hline$N \backslash M$ & 2 & 4 & 8 & 16 & 32 \\
\hline $2|\mathscr{A}(m)|$ & 1 & 2 & 2 & 1 & 1 \\
$4|\mathscr{A}(m)|$ & 10 & 10 & 10 & 10 & 10 \\
$8|\mathscr{A}(m)|$ & & & & & \\
\hline
\end{tabular}

Table $3(d, m)=(4,3)$, $|\mathscr{A}(m)|=94$

\begin{tabular}{llllll}
\hline$N \backslash M$ & 2 & 4 & 8 & 16 & 32 \\
\hline $2|\mathscr{A}(m)|$ & 2 & 0 & 0 & 2 & 1 \\
$4|\mathscr{A}(m)|$ & 10 & 10 & 10 & 10 & 10 \\
$8|\mathscr{A}(m)|$ & & & & & \\
\hline
\end{tabular}

Table $4(d, m)=(2,5)$, $|\mathscr{A}(m)|=119$

\begin{tabular}{llllll}
\hline$N \backslash M$ & 2 & 4 & 8 & 16 & 32 \\
\hline $2|\mathscr{A}(m)|$ & 0 & 0 & 0 & 0 & 0 \\
$4|\mathscr{A}(m)|$ & 7 & 10 & 10 & 10 & 10 \\
$8|\mathscr{A}(m)|$ & 10 & & & & \\
\hline
\end{tabular}

Table $5(d, m)=(3,5)$, $|\mathscr{A}(m)|=516$

\begin{tabular}{llllll}
\hline$N \backslash M$ & 2 & 4 & 8 & 16 & 32 \\
\hline $2|\mathscr{A}(m)|$ & 0 & 0 & 0 & 0 & 0 \\
$4|\mathscr{A}(m)|$ & 2 & 10 & 10 & 10 & 10 \\
$8|\mathscr{A}(m)|$ & 10 & & & & \\
\hline
\end{tabular}

(see Sect. 3.3). Our objective is to give a construction method for a general setting where there are no limitations on the number of iterations of the integral $(k$ in (2)). For this purpose, we adopt an optimization-based viewpoint instead of algebraic ones and extend to our situation the result of [10], which gives a randomized construction of general cubature formula (Tables 1, 2, 3, 4, 5, and 6).

We should mention the Kusuoka approximation [13, 15, 29], which is closely related to cubature on Wiener space. However, our objective in this paper is limited to the construction of the cubature on Wiener space, and the 
Table $6(d, m)=(2,7)$, $|\mathscr{A}(m)|=696$

\begin{tabular}{llllll}
\hline$N \backslash M$ & 2 & 4 & 8 & 16 & 32 \\
\hline $2|\mathscr{A}(m)|$ & 0 & 0 & 0 & 0 & 0 \\
$4|\mathscr{A}(m)|$ & 0 & 0 & 0 & 1 & 8 \\
$8|\mathscr{A}(m)|$ & 0 & 0 & 10 & 10 & 10 \\
\hline
\end{tabular}

optimization-based approach to the general Kusuoka approximation is deferred for future work.

\subsection{Monte Carlo approach to generalized cubature}

A cubature formula is originally a numerical integration formula on some Euclidean space that exactly integrates polynomials up to a certain degree [30], the theory of which underlies cubature on Wiener space introduced in the previous section. Then, we shall explain it in a generalized setting and briefly explain the idea of [10] for constructing general cubature formulas.

Let $(\Omega, \mathscr{G})$ be some measurable space and $X$ be a random variable on it. A generalized cubature formula with respect to $X$ and integrable functions $\varphi_{1}, \ldots, \varphi_{D}: \Omega \rightarrow \mathbb{R}$ is a set of points $x_{1}, \ldots, x_{n} \in \Omega$ and positive weights, $\lambda_{1}, \ldots, \lambda_{n}$ such as

$$
\mathrm{E}\left[\varphi_{i}(X)\right]=\sum_{j=1}^{n} \lambda_{j} \varphi_{i}\left(x_{j}\right), \quad i=1, \ldots, D .
$$

For simplicity, we then assume $\varphi_{1} \equiv 1$. In this setting, $\lambda_{1}+\cdots+\lambda_{n}=1$ must hold. We can also regard the above condition as one vector-valued equality $\mathrm{E}[\boldsymbol{\varphi}(X)]=\sum_{j=1}^{n} \lambda_{j} \varphi\left(x_{j}\right)$, where $\boldsymbol{\varphi}: \Omega \rightarrow \mathbb{R}^{D}$ is defined as $\boldsymbol{\varphi}=\left(\varphi_{1}, \ldots, \varphi_{D}\right)^{\top}$. The existence of such formula is assured by the following theorem [2, 28, 31]:

Theorem 1 (Generalized Tchakaloff's theorem) Under the above setting, there exists a cubature formula whose number of points satisfies $n \leq D$. Moreover, we can take points $x_{1}, \ldots, x_{n}$ so as to satisfy $\varphi\left(x_{j}\right) \in \operatorname{supp} \mathrm{P}_{\varphi(X)}$ for each $j=1, \ldots, n$.

In the above statement, $\operatorname{supp} \mathrm{P}_{\varphi(X)}$ is the support of the distribution of the vector-valued random variable $\varphi(X)$. Equivalently, this is the smallest closed set $A \subset \mathbb{R}^{D}$ satisfying $\mathrm{P}(\varphi(X) \in A)=1$. Generalized Tchakaloff's theorem can be understood as an immediate consequence of a discrete-geometric argument. Indeed, $\mathrm{E}[\varphi(X)]$ is contained in the convex hull of supp $\mathrm{P}_{\varphi(X)}$ (the convex hull of an $A \subset \mathbb{R}^{D}$ is defined as conv $\left.A:=\left\{\sum_{i=1}^{m} \lambda_{i} x_{i} \mid m \geq 1, \lambda_{i} \geq 0, \sum_{i=1}^{m} \lambda_{i}=1, x_{i} \in A\right\}\right)$. Therefore, the generalized Tchakaloff's theorem follows Carathéodory's theorem (note that we assume $\varphi_{1} \equiv 1$ ):

Theorem 2 (Carathéodory) For an arbitrary $A \subset \mathbb{R}^{D}$ and $x \in \operatorname{conv} A$, there exists $D+1$ points $x_{1}, \ldots, x_{D+1} \in A$ such that $x \in \operatorname{conv}\left\{x_{1}, \ldots, x_{D+1}\right\}$. 
Although the above argument cannot directly be used in the construction of cubature, we can use its nature by introducing the concept of relative interior. For a set $A \subset \mathbb{R}^{D}$, its affine hull is defined by

$$
\operatorname{aff} A:=\left\{\sum_{i=1}^{m} \lambda_{i} x_{i} \mid m \geq 1, \lambda_{i} \in \mathbb{R}, \sum_{i=1}^{m} \lambda_{i}=1, x_{i} \in A\right\}
$$

Then, the relative interior of $A$ is the interior of $A$ regarding the subspace topology on aff $A$ and denoted by $\operatorname{ri} A$. In terms of this relative interior, the following generalization of Carathéodory's theorem holds:

Theorem 3 ([3, 30]) Suppose an $A \subset \mathbb{R}^{D}$ satisfies aff $A$ is a $k$-dimensional affine subspace of $\mathbb{R}^{D}$. Then, for each $x \in \mathrm{ri}$ conv $A$, there exists some subset $B \subset A$ composed of at most $2 k$ points such that $\operatorname{aff} B=\operatorname{aff} A$ and $x \in \operatorname{ri} \operatorname{conv} B$.

From this generalization and the fact that

$$
\mathrm{E}[\varphi(X)] \in \operatorname{ri} \operatorname{conv} \operatorname{supp} \mathrm{P}_{\varphi(X)}
$$

holds (see, e.g., [10] or essentially [2] for proof), we obtain the following randomized construction of cubature formulas from i.i.d. copies of $X$ :

Theorem 4 ([10]) Let $X_{1}, X_{2}, \ldots$ be i.i.d. copies of $X$. Then there exists almost surely a positive integer $n$ satisfying $\mathrm{E}[\varphi(X)] \in \operatorname{conv}\left\{\varphi\left(X_{1}\right), \ldots, \varphi\left(X_{n}\right)\right\}$.

Though the weights remain undetermined, for a sufficiently large $n$, it suffices to take a basic feasible solution of the linear programming problem

$$
\text { minimize } 0 \text { subject to } \sum_{j=1}^{n} \lambda_{j} \varphi\left(X_{j}\right)=\mathrm{E}[\varphi(X)], \lambda_{j} \geq 0 .
$$

Indeed, its basic feasible solution satisfies the bound of points used in a cubature given in Tchakaloff's theorem (Theorem 1). This sort of technique reducing the number of points in a discrete measure is called Carathéodory-Tchakaloff subsampling [25].

Here, if we formally write the iterated integral $\int_{0<t_{1}<\cdots<t_{k}<T} \mathrm{~d} w^{i_{1}}\left(t_{1}\right) \cdots \mathrm{d} w^{i_{k}}\left(t_{k}\right)$ appearing in (2) as $\varphi_{\left(i_{1}, \ldots, i_{k}\right)}(w)$ for a valid $w$ (and the Brownian motion $B$ ), then the cubature on Wiener space is a set of paths $w_{j}$ and weights $\lambda_{j}$ formally satisfying

$$
\mathrm{E}\left[\boldsymbol{\varphi}_{\mathrm{W}}(B)\right]=\sum_{j=1}^{n} \lambda_{j} \boldsymbol{\varphi}_{\mathrm{W}}\left(w_{j}\right)
$$

where $\varphi_{\mathrm{W}}$ denotes a vector of some functions of the form $\varphi_{\left(i_{1}, \ldots, i_{k}\right)}$. Therefore, if we could directly generate sample paths of the Brownian motion, then Theorem 4 should be applicable. In reality, it is impossible to generate a Brownian motion on a 
computer, and it is not even a BV path. However, we assume the following variant, supporting our arguments.

Remark 1 The assumption that $X_{1}, X_{2}, \ldots$ possess the same distribution as $X$ in Theorem 4 can be relaxed; the same conclusion yields from the following condition for the i.i.d. sequence:

$$
\text { aff } \operatorname{supp} \mathrm{P}_{\varphi\left(X_{1}\right)}=\operatorname{aff} \operatorname{supp} \mathrm{P}_{\varphi(X)}, \quad \operatorname{supp} \mathrm{P}_{\varphi\left(X_{1}\right)} \supset \operatorname{supp} \mathrm{P}_{\varphi(X)} .
$$

From this fact, it is sufficient to investigate the distribution of iterated integrals, and we indeed show the Wiener space counterpart of the condition (5) in Proposition 15.

\section{Theoretical background of cubature on Wiener space}

In this section, we provide a theoretical review on the cubature theory on Wiener space, first introduced by [19]. We quickly introduce basic notions concerning multidimensional stochastic flows, and give the error estimate of cubature formula. Moreover, we will demonstrate few examples of concrete construction of cubature formula on Wiener space in Sect. 3.3.

\subsection{Vector fields}

In this section, we define vector fields on $\mathbb{R}^{N}$ and show the correspondence between vector fields and vector-valued functions. Let $C^{\infty}\left(\mathbb{R}^{N}\right)$ be the set of real-valued smooth functions over $\mathbb{R}^{N}$.

Definition 5 A vector field on $\mathbb{R}^{N}$ is a $\left(\mathbb{R}\right.$-)linear mapping $V: C^{\infty}\left(\mathbb{R}^{N}\right) \rightarrow C^{\infty}\left(\mathbb{R}^{N}\right)$ such that $V(f g)=(V f) g+f V g$ holds for arbitrary $f, g \in C^{\infty}\left(\mathbb{R}^{N}\right)$.

Due to this condition, a vector field on $\mathbb{R}^{N}$ has to be a differential operator $\sum_{i=1}^{N} V^{i} \partial_{i}$ where $V^{i} \in C^{\infty}\left(\mathbb{R}^{N}\right)$ and $\partial_{i}$ denotes the $i$-th partial derivative for $i=1, \ldots, d$. Therefore, a vector field corresponds to the vector-valued smooth function $\left(V^{1}, \ldots, V^{N}\right)^{\top}: \mathbb{R}^{N} \rightarrow \mathbb{R}^{N}$. By abuse of notation, we also denote this vectorvalued function by $V$.

If $A$ and $B$ are vector fields on $\mathbb{R}^{N}$, we define the Lie bracket $[A, B]:=A B-B A$. This $[A, B]$ is also a vector field because the second derivatives vanish. Note that $[A, B]$ corresponds to the vector $(\partial B) A-(\partial A) B$, where $A, B$ are regarded as functions and $\partial C$ denotes the Jacobian matrix of $C$ (see, e.g., [9]).

Reciprocally, the coefficients of the SDE (1) can be regarded as vector fields. These vector fields are closely related to the behavior of $X_{t}$.

Let $V_{0}, \ldots, V_{d}$ be the vector fields (operators) induced by the coefficients of (1), and define the operator $L:=V_{0}+\frac{1}{2}\left(V_{1}^{2}+\cdots+V_{d}^{2}\right)$. Let us consider the parabolic partial differential equation (PDE) 


$$
\left\{\begin{array}{l}
\frac{\partial}{\partial t} u(t, x)=L u(t, x) \\
u(0, x)=f(x)
\end{array}\right.
$$

with a Lipschitz function $f: \mathbb{R}^{N} \rightarrow \mathbb{R}^{N}$. Because $u(T, x)=\mathrm{E}\left[f\left(X_{T}(x)\right)\right]$ holds [11], we can exploit the numerical schemes in PDE theory to get $\mathrm{E}\left[f\left(X_{T}(x)\right)\right]$ and vice versa.

We also introduce several conditions on the vector fields. They are assumed to obtain the estimate given in the Proposition 6. Before we state those, we introduce some notations based on [13].

Let $\mathscr{A}:=\{\emptyset\} \cup \bigcup_{k=1}^{\infty}\{0,1, \ldots, d\}^{k}$. For $\alpha \in \mathscr{A}$, define $|\alpha|:=0$ if $\alpha=\emptyset$ and $|\alpha|:=k$ if $\alpha=\left(\alpha_{1}, \ldots, \alpha_{k}\right) \in\{0, \ldots, d\}^{k}$. We also define $\|\alpha\|:=|\alpha|+\left|\left\{1 \leq j \leq|\alpha| \mid \alpha_{j}=0\right\}\right|$. For $\alpha, \beta \in \mathscr{A}$, define $\alpha * \beta:=\left(\alpha_{1}, \ldots, \alpha_{|\alpha|}, \beta_{1}, \ldots, \beta_{|\beta|}\right)$. Let $\mathscr{A}_{0}:=\mathscr{A} \backslash\{\emptyset\}$ and $\mathscr{A}_{1}:=\mathscr{A} \backslash\{\emptyset \cup(0)\}$. We also define, for each integer $m \geq 1$,

$$
\mathscr{A}(m):=\{\alpha \in \mathscr{A} \mid\|\alpha\| \leq m\}, \quad \mathscr{A}_{0}(m):=\mathscr{A}(m) \cap \mathscr{A}_{0}, \quad \mathscr{A}_{1}(m):=\mathscr{A}(m) \cap \mathscr{A}_{1} .
$$

Define a vector field $V_{[\alpha]}$ for each $\alpha \in \mathscr{A}$ inductively by $V_{[\emptyset]}=0$ and

$$
\begin{array}{ll}
V_{[i]}\left(=V_{[(i)]}\right):=V_{i} & (i=0, \ldots, d), \\
V_{[\alpha *(i)]}:=\left[V_{\alpha}, V_{i}\right] & (|\alpha| \geq 1, i=0, \ldots, d) .
\end{array}
$$

We can now state the uniformly finitely generated (UFG) condition $[13,16]$ :

(UFG) There exists a positive integer $L \geq 1$ such that, for an arbitrary $\alpha \in \mathscr{A}_{1}$, there exists $\varphi_{\alpha, \beta} \in C_{b}^{\infty}\left(\mathbb{R}^{N}\right)$ for each $\beta \in \mathscr{A}_{1}(L)$ satisfying

$$
V_{[\alpha]}=\sum_{\beta \in \mathscr{A}_{1}(L)} \varphi_{\alpha, \beta} V_{[\beta]} .
$$

This is equivalent to the statement that the $C_{b}^{\infty}\left(\mathbb{R}^{N}\right)$-module generated by $\left\{V_{[\alpha]} \mid \alpha \in \mathscr{A}_{1}\right\}$ is finitely generated. Note that (UFG) is known to be strictly weaker than the uniform Hörmander condition (see, e.g., Example 2 in [14]), which is one of the typical assumptions on vector fields.

Although only the condition (UFG) was assumed in [19], it was pointed out in [5] that the following condition is also essential:

(V0) There exists $\varphi_{\beta} \in C_{b}^{\infty}\left(\mathbb{R}^{N}\right)$ for each $\beta \in \mathscr{A}_{1}(2)$ such that

$$
V_{0}=\sum_{\beta \in \mathscr{A}_{1}(2)} \varphi_{\beta} V_{[\beta]} .
$$

For a function $f \in C_{b}^{\infty}\left(\mathbb{R}^{N}\right)$, define $\left(P_{t} f\right)(x):=\mathrm{E}\left[f\left(X_{t}(x)\right)\right]$. The following estimate is essential.

Proposition 6 ([5, 16]) Assume that both (UFG) and (V0) hold. Then, for any positive integer $r$ and $\alpha_{1}, \ldots, \alpha_{r} \in \mathscr{A}$, there exists a constant $C>0$ such that 


$$
\left\|V_{\left[\alpha_{1}\right]} \cdots V_{\left[\alpha_{r}\right]} P_{t} f\right\|_{\infty} \leq \frac{C t^{1 / 2}}{t^{\left(\left\|\alpha_{1}\right\|+\cdots+\left\|\alpha_{r}\right\|\right) / 2}}\|\nabla f\|_{\infty}
$$

Although we can obtain a weaker bound without assuming (V0), we later exploit this bound assuming both (UFG) and (V0) for simplicity.

We finally state the stochastic Taylor formula in terms of the vector-field notation introduced above. By Itô's formula, we obtain for any $f \in C_{b}^{\infty}\left(\mathbb{R}^{N}\right)$

$$
\begin{aligned}
f\left(X_{t}\right) & =f(x)+\sum_{i=0}^{d} \sum_{j=1}^{N} \int_{0}^{t} V_{i}^{j}\left(X_{s}\right) \partial_{j} f\left(X_{s}\right) \circ \mathrm{d} B_{s}^{i} \\
& =f(x)+\sum_{i=0}^{d} \int_{0}^{t}\left(V_{i} f\right)\left(X_{s}\right) \circ \mathrm{d} B_{s}^{i},
\end{aligned}
$$

where we denote $\mathrm{d} s$ by $\circ \mathrm{d} B_{s}^{0}$. Therefore, the repetition of Ito's formula yields

$$
f\left(X_{t}\right)=f(x)+\sum_{i=0}^{d}\left(V_{i} f\right)(x) \int_{0<s<t} \circ \mathrm{d} B_{s}^{i}+\sum_{i, j=0}^{d}\left(V_{i} V_{j} f\right)(x) \int_{0<t_{1}<t_{2}<1} \circ \mathrm{d} B_{t_{1}}^{i} \circ \mathrm{d} B_{t_{2}}^{j} \cdots .
$$

This is the stochastic Taylor formula, which is rigorously stated as follows. For a multiindex $\alpha=\left(\alpha^{1}, \ldots, \alpha^{k}\right) \in \mathscr{A}$, we denote by $V_{\alpha}$ the operator $V_{\alpha^{1}} \cdots V_{\alpha^{k}}$.

Proposition 7 ([19, Proposition 2.1]; [12]) Let $f \in C_{b}^{\infty}\left(\mathbb{R}^{N}\right)$ and $m$ be a positive integer. Then, we have

$$
f\left(X_{t}(x)\right)=\sum_{\alpha \in \mathscr{A}(m)}\left(V_{\alpha} f\right)(x)+R_{m}(t, x, f),
$$

where the remainder term satisfies, for some constant $C>0$,

$$
\sup _{x \in \mathbb{R}^{N}} \sqrt{\mathrm{E}\left[R_{m}(t, x, f)^{2}\right]} \leq C t^{\frac{m+1}{2}} \sup _{\beta \in \mathscr{A}(m+2) \backslash \mathscr{A}(m)}\left\|V_{\beta} f\right\|_{\infty} .
$$

\subsection{Formulation and evaluation of cubature on Wiener space}

We can now precisely define the cubature formula [19].

Definition 8 Let $T>0$, and let $m$ be a positive integer. BV paths $w_{1}, \ldots, w_{n} \in C_{0}^{0}\left([0, T] ; \mathbb{R} \oplus \mathbb{R}^{d}\right)$ and weights $\lambda_{1}, \ldots, \lambda_{n} \geq 0$ with $\sum_{i=1}^{n} \lambda_{j}=1$ to define a cubature formula on Wiener space of degree $m$ at time $T$, if only

$$
\mathrm{E}\left[\int_{0<t_{1}<\cdots<t_{k}<T} \circ \mathrm{d} B_{t_{1}}^{i_{1}} \cdots \circ \mathrm{d} B_{t_{k}}^{i_{k}}\right]=\sum_{j=1}^{n} \lambda_{j} \int_{0<t_{1}<\cdots<t_{k}<T} \mathrm{~d} w_{j}^{i_{1}}\left(t_{1}\right) \cdots \mathrm{d} w_{j}^{i_{k}}\left(t_{k}\right) .
$$

holds for all $\left(i_{1}, \ldots, i_{k}\right) \in \mathscr{A}(m)$. Here, o $\mathrm{d} B_{s}^{0}$ represents $\mathrm{d} s$. 
To construct a cubature formula, it suffices to find it over [0, 1]. Indeed, when $w_{1}, \ldots, w_{n} \in C_{0}^{0}\left([0,1] ; \mathbb{R} \oplus \mathbb{R}^{d}\right)$ form the cubature over $[0,1]$,

$$
w_{T, j}^{i}(t):= \begin{cases}T w_{j}^{i}(t / T) & (i=0) \\ \sqrt{T} w_{j}^{i}(t / T) & (i=1, \ldots, d)\end{cases}
$$

with the same weights define the cubature over $[0, T]$. This is an immediate consequence of the scaling property of the Brownian motion.

Once such paths are given, we can easily compute each evolution driven by $w_{i}$ as it is just an ODE. For a BV path $w \in C_{0}^{0}\left([0, T] ; \mathbb{R} \oplus \mathbb{R}^{d}\right)$, define $\tilde{X}_{t}(x, w)$ as the solution of the ODE

$$
\mathrm{d} \tilde{X}_{t}(x, w)=\sum_{i=0}^{d} V_{i}\left(\tilde{X}_{t}(x, w)\right) \mathrm{d} w^{i}(t), \quad \tilde{X}_{0}(x, w)=x .
$$

Then, $\sum_{j=1}^{n} \lambda_{j} f\left(\tilde{X}_{T}\left(x, w_{T, j}\right)\right)$ should approximate well $\mathrm{E}\left[f\left(X_{T}(x)\right)\right]$. Indeed, the estimate in Proposition 7 holds for $t=T$ if we replace the Wiener measure by the discrete measure $\sum_{j=1}^{n} \lambda_{j} \delta_{w_{j}}$. Therefore, by applying Cauchy-Schwarz we obtain the evaluation:

$$
\sup _{x \in \mathbb{R}^{N}}\left|\mathrm{E}\left[f\left(X_{T}(x)\right)\right]-\sum_{j=1}^{n} \lambda_{j} f\left(\tilde{X}_{T}\left(x, w_{T, j}\right)\right)\right| \leq C T^{\frac{m+1}{2}} \sup _{\beta \in \mathscr{A}(m+2) \backslash \mathscr{A}(m)}\left\|V_{\beta} f\right\|_{\infty}
$$

with the constant $C>0$ depending only on $w_{1}, \ldots, w_{n}$.

The above formula does not work as a good approximation unless $T$ is small. Therefore, we divide $[0, T]$ into smaller time intervals as $0=t_{0}<t_{1}<\cdots<t_{k}=T$. If we consider the repeated application of the cubature formula over each subinterval $\left[t_{\ell-1}, t_{\ell}\right]$, we can use

$$
\sum_{j_{1}, \ldots, j_{k}=1}^{n} \lambda_{j_{1}} \cdots \lambda_{j_{k}} f\left(X_{T}\left(x, w_{s_{1}, j_{1}} * \cdots * w_{s_{k}, j_{k}}\right)\right)
$$

where $w * v$ denotes the concatenation of two paths and $s_{\ell}:=t_{\ell}-t_{\ell-1}$ for each $\ell=1, \ldots k$, as an approximation of the expectation $\mathrm{E}\left[f\left(X_{T}(x)\right)\right]$. If we define discrete Markov random variables $Y_{0}, \ldots, Y_{k}$ independent of the Brownian motion as

$$
Y_{0}=x, \quad \mathrm{P}\left(Y_{\ell}=\tilde{X}_{s_{\ell}, y}\left(w_{s_{\ell}, j}\right) \mid Y_{\ell-1}=y\right)=\lambda_{j} \quad(\ell=1, \ldots, k, j=1, \ldots, n),
$$

$\mathrm{E}\left[Y_{k}\right]$ coincides with the approximation (9). Then, combining an estimate for

$$
\sup _{x \in \mathbb{R}^{N}}\left|\mathrm{E}\left[f\left(Y_{k}\right) \mid Y_{0}=x\right]-\mathrm{E}\left[f\left(X_{T}(x)\right)\right]\right|
$$

with Proposition 6, we can prove the following assertion. 
Proposition 9 ( [19, Proposition 3.6]) Let $f$ be a bounded Lipschitz function in $\mathbb{R}^{N}$. Then, under (UFG) and (V0), we have

$$
\sup _{x \in \mathbb{R}^{N}}\left|\mathrm{E}\left[f\left(Y_{k}\right) \mid Y_{0}=x\right]-\mathrm{E}\left[f\left(X_{T}(x)\right)\right]\right| \leq C\|\nabla f\|_{\infty}\left(s_{k}^{1 / 2}+\sum_{\ell=1}^{k-1} \frac{s_{\ell}^{(m+1) / 2}}{\left(T-t_{\ell}\right)^{m / 2}}\right)
$$

for some constant $C>0$, which is dependent only on $m$ and $w_{1}, \ldots, w_{n}$.

The equally spaced partition $t_{\ell}=\ell T / k$ for $\ell=0, \ldots, k$ is not the optimal one in terms of asymptotic error bound with $k \rightarrow \infty$. Consider taking $t_{\ell}=T\left(1-\left(1-\frac{\ell}{k}\right)^{\gamma}\right)$ with a constant $\gamma>0$ independent of $k(\gamma=1$ corresponds to the equally spaced partition). By taking $\gamma>m-1$, we have the following estimate [13]:

$$
\sup _{x \in \mathbb{R}^{N}}\left|\mathrm{E}\left[f\left(Y_{k}\right) \mid Y_{0}=x\right]-\mathrm{E}\left[f\left(X_{T}(x)\right)\right]\right| \leq C k^{-(m-1) / 2}\|\nabla f\|_{\infty} .
$$

Therefore, a cubature formula of degree $m$ with an appropriate time partition achieves the error rate $\mathrm{O}\left(k^{-(m-1) / 2}\right)$ where $k$ is the number of partitions.

Remark 2 If we have to compute all the $k$-times concatenation of a cubature formula composed of $n$ sample paths, we have to solve $\frac{n^{k+1}-1}{n-1}$ ODEs in total [19]. When number of ODEs is too large, we reduce the computational complexity through some Monte Carlo simulation or subsampling method [17, 25, 32]. In this paper, we do not consider efficient implementation of concatenation of cubature formula, but only consider their constructions.

\subsection{Known constructions of cubature on Wiener space}

We should note that some concrete examples of cubature formulas on Wiener space are already known. The simplest case treated in [19] is $m=3$, where we have a cubature formula composed of linear paths (i.e., with only one linear segment).

Let $n:=2^{d}$ and $z_{1}, \ldots, z_{n} \in \mathbb{R}^{d}$ be all the elements of $\{-1,1\}^{d}$. Then, paths

$$
w_{i}(t):=t\left(1, z_{i}\right)=\left(t, t z_{i}^{1}, \ldots, t z_{i}^{d}\right), \quad 0 \leq t \leq 1, \quad i=1, \ldots, n
$$

with weights $\lambda_{1}=\cdots=\lambda_{n}=2^{-d}$ construct a cubature formula with $m=3$. Although $2^{d}$ is much larger than $|\mathscr{A}(3)|=\mathrm{O}\left(d^{3}\right)$, we can reduce the number of paths, e.g., using Carathéodory-Tchakaloff subsampling.

Constructions for the case $d$ in general and $m=5$ are also given in [19], where the authors give cubature formula using only $\mathrm{O}\left(d^{3}\right)$ paths. Moreover, [8] constructed higher order cubature formula up to $m=11$, but the construction is limited to one dimensional space-time $(d=1)$. Ref. [21] represents other concrete examples when $m=5$ with general $d$ and the case $(d, m)=(2,7)$.

All the aforementioned examples are derived by solving equations in terms of Lie algebra (see Sect. 4.1), which can be directly written using the 
Campbell-Baker-Hausdorff formula. However, as a different approach, we address an optimization-based construction in the next section.

\section{Stochastic Tchakaloff's theorem}

In the previous section, we have demonstrated the theoretical support of cubature on Wiener space. However, it is important to know if such formulas can actually be constructed. In this section, we shall state stochastic Tchakaloff's theorem, which assures the existence of cubature formula on Wiener space. Though the stochastic Tchakaloff's theorem is originally given in [19], we state it in a stronger way by using the concept of relative interior.

Before doing so, we shall introduce the rich algebraic structures behind the theory of cubature on Wiener space in the following two sections, which are also essential in our proof of stochastic Tchakaloff's theorem.

\subsection{Tensor and Lie algebra}

We introduce a tensor algebra which is suitable to our case $[15,18,19]$. Denote $\mathbb{R} \oplus \mathbb{R}^{d}$ by $E$. Define $U_{0}(E):=\mathbb{R}\left(=: E^{\otimes 0}\right)$. Let $A_{0}:=\mathbb{R}$ and $A_{1}:=\mathbb{R}^{d}$, and define

$$
U_{n}(E):=\underset{\substack{\left(i_{1}, \ldots, i_{k}\right) \in\{0,1\}^{k}, 2 k-\left(i_{1}+\cdots+i_{k}\right)=n}}{\bigoplus} A_{i_{1}} \otimes \cdots \otimes A_{i_{k}}
$$

for each positive integer $n$. Here, the condition for $\left(i_{1}, \ldots, i_{k}\right)$ means that $A_{i_{1}} \otimes \cdots \otimes A_{i_{k}}$ takes all the arrangement of $\mathbb{R}$ and $\mathbb{R}^{d}$ such that $2(\#$ of $\mathbb{R})+\left(\#\right.$ of $\left.\mathbb{R}^{d}\right)=n$. Then, we consider the tensor algebra of formal series

$$
T((E)):=\overline{\bigoplus_{n=0}^{\infty} U_{n}}\left(\simeq \overline{\bigoplus_{n=0}^{\infty} E^{\otimes n}}\right),
$$

where the completion (overline) is taken so that $T((E))$ consists of series, i.e., $T((E))$ is the set of all the infinite sequences $\left(a_{n}\right)_{n=0}^{\infty}$ where $a_{n} \in U_{n}$ for each $n \geq 0$. Let $T^{(n)}(E):=\bigoplus_{k=0}^{n} U_{k}$, and let $\pi_{n}: T((E)) \rightarrow T^{(n)}(E)$ be the canonical projection for each $n \geq 0$. As we are only interested in these projections in practice, we do not need to differentiate the usual tensor algebra from that of series (i.e., its completion) treated here [27].

It might be easier to understand $T$ as the ring of formal power series $\mathbb{R}\left[\left[Z_{0}, Z_{1}, \ldots, Z_{d}\right]\right]$ with noncommutative variables $Z_{0}, \ldots, Z_{d} \quad$ (see, e.g., [1]). In that case, we redefine the degree of some monomial $Y$ by $\operatorname{deg} Y:=2 \operatorname{deg}_{Z_{0}} Y+\left(\operatorname{deg}_{Z_{1}} Y+\cdots+\operatorname{deg}_{Z_{d}} Y\right.$ ) (where each $\operatorname{deg}_{Z_{i}} Y$ denotes the number of $Z_{i}$ appearing in $Y$ ) and regard $U_{n}(E)$ as the subspace spanned by monomials of degree $n$ for each $n \geq 0$. 
For any elements $a=\left(a_{n}\right)_{n=0}^{\infty}, b=\left(b_{n}\right)_{n=0}^{\infty} \in T((E))$, we define the sum and product as follows:

$$
a+b:=\left(a_{n}+b_{n}\right)_{n=0}^{\infty}, \quad a \otimes b:=\left(\sum_{i=0}^{n} a_{i} \otimes b_{n-i}\right)_{n=0}^{\infty} .
$$

The action by scalar is element-wise. These definitions are straightforward if we consider $\mathbb{R}\left[\left[Z_{0}, Z_{1}, \ldots, Z_{d}\right]\right]$. Moreover, we define the exponential, inverse, and logarithm:

$$
\exp (a):=\sum_{k=0}^{\infty} \frac{a^{\otimes k}}{k !}, \quad a^{-1}:=\frac{1}{a_{0}} \sum_{k=0}^{\infty}\left(1-\frac{a}{a_{0}}\right)^{\otimes k}, \quad \log a:=\log a_{0}-\sum_{k=1}^{\infty} \frac{1}{k}\left(1-\frac{a}{a_{0}}\right)^{\otimes k},
$$

where the latter two operations are limited for $a \in T((E))$ with $a_{0} \neq 0$. Note that these operations commute with each projection homomorphism $\pi_{n}$.

Let us introduce the space of Lie series. Define

$$
L((E)):=0 \oplus E \oplus[E, E] \oplus[E,[E, E]] \oplus \cdots \subset T((E)) \simeq \overline{\bigoplus_{n=0}^{\infty} E^{\otimes n}},
$$

where, for linear subspaces $A, B \in T((E)),[A, B]$ is the linear subspace of $T((E))$ spanned by Lie brackets $[a, b]:=a \otimes b-b \otimes a(a \in A, b \in B) . L((E))$ is the socalled free Lie algebra generated by $E$ [26]. The elements of $L((E))$ are called Lie series. We also define $L^{(n)}(E):=\pi_{n}(L((E)))$, the elements of which are called Lie polynomials.

\subsection{Signature of a path}

We shall introduce the signature (or Chen series [4]) of a path, which summarizes the algebraic structure of iterated integrals. Let $w=\left(w^{0}, \ldots, w^{d}\right) \in C_{0}^{0}\left([0, T] ; \mathbb{R} \oplus \mathbb{R}^{d}\right)$ be a BV path and we define its signature.

Definition 10 For $0 \leq s \leq t \leq T$, define $S(w)_{s, t} \in T((E)) \simeq \mathbb{R}\left[\left[Z_{0}, Z_{1}, \ldots, Z_{d}\right]\right]$ by

$$
\begin{aligned}
S(w)_{s, t} & :=\sum_{n=0}^{\infty} \int_{s<t_{1}<\cdots<t_{n}<t} \mathrm{~d} w\left(t_{1}\right) \otimes \cdots \otimes \mathrm{d} w\left(t_{n}\right) \\
& :=\sum_{n=0}^{\infty} \sum_{\left(i_{1}, \ldots, i_{k}\right) \in \mathscr{A}(n) \backslash \mathscr{A}(n-1)}\left(\int_{s<t_{1}<\cdots<t_{n}<t} \mathrm{~d} w^{i_{1}}\left(t_{1}\right) \cdots \mathrm{d} w^{i_{k}}\left(t_{n}\right)\right) Z_{i_{1}} \cdots Z_{i_{k}},
\end{aligned}
$$

where the integration by $\mathrm{d} w$ means the Lebesgue-Stieltjes integration. In both presentations, we think of the 0 -th (or constant) term of $S(w)_{s, t}$ as 1 . We call $S(w)_{s, t}$ the signature of $w$ over $[s, t]$.

The following is Chen's theorem. 
Theorem 11 ( $[4,18])$ The process $S(w)$ satisfies $S(w)_{s, t} \otimes S(w)_{t, u}=S(w)_{s, u}$ for arbitrary $0 \leq s \leq t \leq u \leq T$. It also holds that $\log S(w)_{s, t} \in L((E))$ and therefore $\pi_{n}\left(\log S(w)_{s, t}\right) \in L^{(n)}(E)$.

Moreover, the inverse of this correspondence holds, i.e., for an arbitrary Lie polynomial $\mathscr{L} \in L^{(n)}(E) \subset T(E)$ and arbitrary $0 \leq s<t \leq T$, there exists a boundedvariation path $w \in C_{0}^{0}\left([0, T] ; \mathbb{R} \oplus \mathbb{R}^{d}\right)$ such that $\pi_{n}\left(\log S(w)_{s, t}\right)=\mathscr{L}$.

Remark 3 Regarding the latter part, a stronger result is known [7, Theorem 7.28]. Every Lie polynomial can be exactly (not approximately) represented as a (truncated) logarithm of some continuous piecewise linear path with a finite number of linear intervals.

By virtue of these assertions, we see that the problem of finding paths constructing a cubature formula is equivalent to the problem of finding the corresponding Lie polynomials. The following Brownian-motion version of this result is also important.

Proposition 12 ([15, 19]) Define the (Stratonovich) signature of the Brownian motion as an element of $T((E))=\mathbb{R}\left[\left[Z_{0}, Z_{1}, \ldots, Z_{d}\right]\right]$ by

$$
S(B)_{s, t}=\sum_{n=0}^{\infty} \sum_{\left(i_{1}, \ldots, i_{k}\right) \in \mathscr{A}(n) \backslash \mathscr{A}(n-1)}\left(\int_{s<t_{1}<\cdots<t_{n}<t} \circ \mathrm{d} B_{t_{1}}^{i_{1}} \cdots \circ \mathrm{od} B_{t_{n}}^{i_{k}}\right) Z_{i_{1}} \cdots Z_{i_{k}}
$$

for each $0 \leq s \leq t$. Then, $\log S(B)_{s, t}$ is almost surely a Lie series.

As we mainly deal with the signature over [0, 1], hereafter let $S(w)$ and $S(B)$ represent $S(w)_{0,1}$ and $S(B)_{0,1}$, respectively. We also define for each $\alpha=\left(i_{1}, \ldots, i_{k}\right) \in \mathscr{A}$,

$$
I^{\alpha}(w):=\int_{0<t_{1}<\cdots<t_{k}<1} \mathrm{~d} w^{i_{1}}\left(t_{1}\right) \cdots \mathrm{d} w^{i_{k}}\left(t_{k}\right), \quad I^{\alpha}(B):=\int_{0<t_{1}<\cdots<t_{k}<1} \circ \mathrm{d} B_{t_{1}}^{i_{1}} \cdots \circ \mathrm{d} B_{t_{k}}^{i_{k}} .
$$

Note that we set $I^{\alpha}(w)=I^{\alpha}(B)=1$ if $\alpha=\emptyset$.

If to define $\mathscr{L}:=\pi_{n}(\log S(B))$, Indeed, we obtain the expression

$$
\mathrm{E}\left[\pi_{n}(S(B))\right]=\mathrm{E}\left[\pi_{n}(\exp \mathscr{L})\right] .
$$

As $\mathscr{L}$ is a random Lie polynomial from the previous assertion, roughly speaking, the generalized Tchakaloff's theorem (Theorem 1) and the inverse statement in Theorem 11 yield the existence of a cubature formula on Wiener space. However, we should point out that the surjectivity stated in Theorem 11 fails if we require that $w^{0}(t)$ is monotone (so the original proof of stochastic Tchakaloff's theorem in [19] should be modified). 
Proposition 13 Let $n \geq 4$. Then, there exists a Lie polynomial $\mathscr{L} \in L^{(n)}(\mathbb{R} \oplus \mathbb{R})$ such that $\pi_{n}(\exp \mathscr{L})$ cannot be expressed as $\pi_{n}\left(S(w)_{s, t}\right)$ for any $B V$ path $w \in C_{0}^{0}([0, T] ; \mathbb{R} \oplus \mathbb{R})$ with strictly monotone $w^{0}$.

Proof Consider an $\mathbb{R} \oplus \mathbb{R}$-valued continuous BV path $w=\left(w^{0}, w^{1}\right)$ on $[0, T]$ that starts at the origin with $w^{0}$ strictly increasing. We have

$$
S(w)_{0, T}^{(1,1,0)}=\int_{0<t_{1}<t_{2}<t_{3}<T} \mathrm{~d} w^{1}\left(t_{1}\right) \mathrm{d} w^{1}\left(t_{2}\right) \mathrm{d} w^{0}\left(t_{3}\right)=\int_{0}^{T} \frac{w^{1}(t)^{2}}{2} \mathrm{~d} w^{0}(t) .
$$

Because $w^{0}$ is strictly increasing, there exists a differentiation $\frac{\mathrm{d} w^{0}(t)}{\mathrm{d} t} \in L^{1}([0, T])$ that is positive almost everywhere on $[0, T]$. Therefore, if $S(w)_{0, T}^{(1,1,0)} \stackrel{\mathrm{d} t}{=} 0$ holds, then $w^{1}$ is zero almost everywhere and so $S(w)_{0, T}^{(1,0)}=0$ holds in particular. We have the same conclusion for strictly decreasing $w^{0}$, so, we have

$$
S(w)_{0, T}^{(1,1,0)}=0 \quad \Longrightarrow \quad S(w)_{0, T}^{(1,0)}=0
$$

for each $w=\left(w^{0}, w^{1}\right)$ with strictly monotone $w^{0}$.

Let $e_{0}, e_{1} \in \mathbb{R} \oplus \mathbb{R}$ be the standard basis. If we consider

$$
Y:=\exp \left(e_{0}+\left[e_{0}, e_{1}\right]\right)=\sum_{i=0}^{\infty} \frac{1}{n !}\left(e_{0}+e_{0} \otimes e_{1}-e_{1} \otimes e_{0}\right)^{\otimes n} \in T((\mathbb{R} \oplus \mathbb{R})),
$$

then its coefficient of $e_{1} \otimes e_{1} \otimes e_{0}$ is obviously zero, whereas that of $e_{1} \otimes e_{0}$ is -1 . As $e_{0}+\left[e_{0}, e_{1}\right]$ is clearly a Lie polynomial, the proof is complete.

Although the surjectivity fails, we can actually prove the existence of a cubature formula with $w^{0}(t)=t$ in the following section. We use the following wellknown approximation statement for the Brownian motion.

Proposition 14 Let $n$ be a positive integer and $B$ be $a$ d-dimensional Brownian motion. Then, with probability one, the sequence of piecewise linear paths $w_{1}, w_{2}, \cdots \in C_{0}^{0}\left([0,1] ; \mathbb{R} \oplus \mathbb{R}^{d}\right)$ given by linearly interpolating $w_{k}\left(j / 2^{k}\right)=B\left(j / 2^{k}\right)$ for $j=0,1, \ldots, 2^{k}$ satisfies

$$
\pi_{n}\left(S\left(w_{k}\right)\right) \rightarrow \pi_{n}(S(B)), \quad k \rightarrow \infty .
$$

Proof We only give a sketch as we use arguments based on rough paths. If there is no time-term (the zero-th entry of the path) and $n=2$, then the result yields from the well-known dyadic piecewise-linear approximation for the Brownian rough path (see [6, Proposition 3.6] or [7, Proposition 13.18]). Adding the time (zero-th entry) is not difficult as it is sufficiently smooth and does not affect the regularity of the rough path. To generalize $n$ from $n=2$, it suffices to observe the continuity of the "Lyons lift" (also see [7, Chapter 9]). 


\subsection{Proof of stochastic Tchakaloff's theorem}

Throughout the section, we fix a positive integer $m$ and consider elements in $T^{(m)}(E)$. Note that $T^{(m)}(E)$ can naturally be regarded in the same light as $F:=\mathbb{R}^{\mathscr{A}(m)}$. Define a set $G$ (as a subset of $F$ ) by

$$
G:=\left\{S(w) \mid w \in C_{0}^{0}\left([0,1] ; \mathbb{R} \oplus \mathbb{R}^{d}\right) \text { is a BV path, } w^{0}(1)=1\right\}
$$

From Theorem 11, this coincides with the set of $\exp (\mathscr{L})$, where $\mathscr{L}$ is a Lie polynomial such that the coefficient of $Z_{0}$ is 1 .

We denote the distribution of $S(B)$ over $F$ by $\mathrm{P}_{S(B)}$. We shall argue the relation of $G$ and supp $\mathrm{P}_{S(B)}$ in the following.

Proposition 15 It holds that aff $\operatorname{supp} \mathrm{P}_{S(B)}=\operatorname{aff} G$.

Proof From Proposition 12, supp $\mathrm{P}_{S(B)} \subset$ aff $G$ holds (as aff $G$ includes the closure of $G$ ). Therefore, it is sufficient to show $G \subset$ aff $\operatorname{supp} \mathrm{P}_{S(B)}$.

As aff supp $\mathrm{P}_{S(B)}$ is the intersection of all the hyperplanes, which includes supp $\mathrm{P}_{S(B)}$, it can be represented as

$$
\text { aff } \operatorname{supp} \mathrm{P}_{S(B)}=\bigcap_{(c, d) \in H}\left\{\boldsymbol{v} \in F \mid \boldsymbol{c}^{\top} \boldsymbol{v}=d\right\},
$$

where $H$ is the family of all $(\boldsymbol{c}, d) \in F \times \mathbb{R}$ such that $\boldsymbol{c}^{\top} S(B)=d$ holds almost surely. The problem is now reduced to the statement

$$
c^{\top} S(B)=d \text { a.s. } \quad \Longrightarrow \quad c^{\top} S(w)=d
$$

for every $w$ appearing in the definition of $G$. This results from the following lemma as $\pi_{0}(S(B))=\pi_{0}(S(w))=1$ always holds.

The following is the key lemma in the above proof. We give its proof in the appendix as it is elementary.

Lemma 16 Let $\left(c_{\alpha}\right)_{\alpha \in \mathscr{A}} \in \mathbb{R}^{\mathscr{A}}$ be a vector whose all but finite entries are zero. Then, if $\sum_{\alpha \in \mathscr{A}} c_{\alpha} I^{\alpha}(B)=0$ holds almost surely,

$$
\sum_{\alpha \in \mathscr{A}} c_{\alpha} I^{\alpha}(w)=0
$$

holds for every bounded-variation path $w \in C_{0}^{0}\left([0,1] ; \mathbb{R} \oplus \mathbb{R}^{d}\right)$ with $w^{0}(1)=1$.

The following is a stochastic version of Tchakaloff's theorem, which assures the existence of cubature formulas on Wiener space. It is stated in a little stronger way than [19, Theorem 2.4], wherein the "relative interior" did not appear. Note that the time interval considered hereafter is $[0,1]$. 
Theorem 17 Let $m$ be a positive integer. There exist $n B V$ paths $w_{1}, \ldots, w_{n} \in C_{0}^{0}\left(\mathbb{R} \oplus \mathbb{R}^{d}\right)$ and $n$ positive weights $\lambda_{1}, \ldots, \lambda_{n}$ whose sum is 1 that satisfy $n \leq|\mathscr{A}(m)|$ and

$$
\mathrm{E}\left[\pi_{m}(S(B))\right]=\sum_{i=1}^{n} \lambda_{i} \pi_{m}\left(S\left(w_{i}\right)\right) .
$$

Moreover, if we loosen the condition to be $n \leq 2|\mathscr{A}(m)|, w_{1}, \ldots, w_{n}$ can be taken such that $\pi_{m}\left(S\left(w_{i}\right)\right)$ is contained in $G$ for each $i$, aff $\left\{\pi_{m}\left(S\left(w_{1}\right)\right), \ldots, \pi_{m}\left(S\left(w_{n}\right)\right)\right\}=\operatorname{aff} G$, and

$$
\mathrm{E}\left[\pi_{m}(S(B))\right] \in \text { ri conv }\left\{\pi_{m}\left(S\left(w_{1}\right)\right), \ldots, \pi_{m}\left(S\left(w_{n}\right)\right)\right\} .
$$

Proof By virtue of Carathéodory's theorem,

the former part follows from the latter part. We here show the latter part.

From Theorem 3, (3) and Proposition 15, we can find $n$ Lie polynomials $\mathscr{L}_{1}, \ldots, \mathscr{L}_{n}$ such that each $\pi_{m}\left(\exp \mathscr{L}_{i}\right)$ is contained in $G$, and $\mathrm{E}\left[\pi_{m}(S(B))\right]$ is contained in the relative interior of their convex hull. Here, $n$ can actually be taken such that $n \leq 2 \operatorname{dim} G(\leq 2|\mathscr{A}(m)|)$ because of Theorem 3. From the correspondence stated in Chen's theorem (Theorem 11), we can find a desired set of paths in $C_{0}^{0}\left([0,1] ; \mathbb{R} \oplus \mathbb{R}^{d}\right)$. Note that the condition $\pi_{m}\left(\exp \mathscr{L}_{i}\right) \subset G$ implies that the corresponding path satisfies $w_{i}^{0}(1)=1$.

Remark 4 By exploiting Proposition 14, we can also prove the same result even if we require $w_{i}^{0}(t)=t$ for each $i=1, \ldots, n$ and $0 \leq t \leq 1$. Although Proposition 14 only asserts an approximation result, "relative interior" argument fills the gap.

\section{Monte Carlo approach to cubature on Wiener space}

In this section, we investigate a way to construct cubature formulas, which is based on mathematical optimization instead of Lie algebra. We limit the arguments to cubature formula composed of continuous piecewise linear paths, and propose a construction based on Monte Carlo sampling, which is the application of [10] to our case. We also carry out numerical experiments in concrete cases.

Although existing constructions of cubature formulas on Wiener space are based on Lie-algebraic equations, we can simply regard the cubature construction as an optimization problem. One such way is to consider an LP problem, which is analogous to ordinary cubature problems treated in Sect. 2.2. From this viewpoint, we can naively generate many sample paths and then reduce their number by using Carathéodory-Tchakaloff subsampling. We later see that this approach is applicable at least theoretically (Sect. 5.2). 


\subsection{Signature of continuous BV paths}

In this section, we see the properties of BV paths and their signature. We also see that the truncated signature of continuous BV paths can be approximated with any accuracy by that of piecewise linear paths.

Let $w=\left(w^{0}, w^{1}, \ldots, w^{d}\right) \in C_{0}^{0}\left([0,1] ; \mathbb{R} \oplus \mathbb{R}^{d}\right)$ be BV paths. We define the total variation of $w$ as

$$
\|w\|_{1}:=\sup _{\Delta} \sum_{i=1}^{k} \max _{0 \leq j \leq d}\left|w^{j}\left(t_{i}\right)-w^{j}\left(t_{i-1}\right)\right|\left(=\sup _{\Delta} \sum_{i=1}^{k}\left\|w\left(t_{i}\right)-w\left(t_{i-1}\right)\right\|_{\infty}\right),
$$

where $\Delta$ is the partition of $[0,1]$ by $0=t_{0}<t_{1}<\cdots<t_{k}=1$ and $k$ varies in $\sup _{\Delta}$. We call $w$ a BV path if $\|w\|_{1}<\infty$ holds. Note that other norms are also equivalent as the space $\mathbb{R} \oplus \mathbb{R}^{d}$ is finite-dimensional though we are using the sup-norm $\|\cdot\|_{\infty}$ of $\mathbb{R} \oplus \mathbb{R}^{d}$

We can reparameterize $w$ so that it becomes Lipschitz continuous if necessary. Indeed, if we let $\left\|\left.w\right|_{[s, t]}\right\|_{1}$ be the total variation of $w$ over $[s, t]$ and

$$
\tau(t):=\frac{\left\|\left.w\right|_{[0, t]}\right\|_{1}}{\|w\|_{1}}
$$

for a nonconstant $w$, then $w \circ \tau$ is a well-defined Lipschitz path ( $\tau$ becomes a nondecreasing function onto $[0,1])$. It is also important that the signature is invariant under this reparametrization (see, e.g., [7, Proposition 1.42 and 7.10]; note that $w^{0}(t)=t$ might be lost then even if the original path satisfies it).

Hereafter, we may assume that there exist $d+1$ derivative functions $f^{0}, f^{1}, \ldots, f^{d} \in L^{\infty}([0,1] ; \mathbb{R})$ such that

$$
w^{j}(t)=\int_{0}^{t} f^{j}(s) \mathrm{d} s, \quad t \in[0,1], j=0,1, \ldots, d .
$$

In this case, the total variation of $w$ can be written as

$$
\|w\|_{1}=\int_{0}^{1} \max _{0 \leq j \leq d}\left|f^{j}(s)\right| \mathrm{d} s .
$$

Signature can also be represented by the derivatives as

$$
\begin{aligned}
I^{\alpha}(w) & =\int_{0<t_{1}<\cdots<t_{k}<1} \mathrm{~d} w^{i_{1}}\left(t_{1}\right) \cdots \mathrm{d} w^{i_{k}}\left(t_{k}\right) \\
& =\int_{0<t_{1}<\cdots<t_{k}<1} f^{i_{1}}\left(t_{1}\right) \cdots f^{i_{k}}\left(t_{k}\right) \mathrm{d} t_{1} \cdots \mathrm{d} t_{k} .
\end{aligned}
$$

for each multiindex $\alpha=\left(i_{1}, \ldots, i_{k}\right) \in \mathscr{A}$.

As a special case of BV paths, we are interested in (continuous) piecewise linear paths, which are easy to implement on computers. Let $0=s_{0}<s_{1}<\cdots<s_{n}=1$ be a partition of $[0,1]$. Then, we can define a path $w \in C_{0}^{0}\left([0,1] ; \mathbb{R} \oplus \mathbb{R}^{d}\right)$ which is 
linear on each interval $\left[s_{j-1}, s_{j}\right](j=1, \ldots, n)$ by determining the slope vector in $\mathbb{R} \oplus \mathbb{R}^{d}$ at each interval. For the sake of computation, here we give the calculation of the signature explicitly. Let $g_{j}=\left(g_{j}^{0}, g_{j}^{1}, \ldots, g_{j}^{d}\right)$ be the slope of $w$ over $\left(s_{j-1}, s_{j}\right)$. Then, for $\alpha=\left(i_{1}, \ldots, i_{k}\right) \in \mathscr{A}$,

$$
I^{\alpha}(w)=\sum_{\substack{1 \leq \ell_{1} \leq \cdots \leq \ell_{n-1} \leq k+1 \\ \ell_{0}=1, \ell_{n}=k+1}} \prod_{j=1}^{n} \frac{\left(s_{j}-s_{j-1}\right)^{\ell_{j}-\ell_{j-1}}}{\left(\ell_{j}-\ell_{j-1}\right) !} \prod_{\ell=\ell_{j-1}}^{\ell_{j}-1} g_{j}^{i_{\ell}}
$$

holds. We can derive this by dividing the integral domain into disjoint segments, which are compatible with the partition $0=s_{0}<s_{1}<\cdots<s_{n}=1$. If we adopt the notation $g_{j}^{\alpha}:=g_{j}^{i_{1}} \cdots g_{j}^{i_{k}}$ for each $\alpha \in \mathscr{A}, I^{\alpha}(w)$ can also be written as

$$
I^{\alpha}(w)=\sum_{\alpha_{1} * \cdots * \alpha_{n}=\alpha} \prod_{j=1}^{n} \frac{\left(s_{j}-s_{j-1}\right)^{\left|\alpha_{j}\right|}}{\left|\alpha_{j}\right| !} g_{j}^{\alpha} .
$$

The latter expression can also be easily derived from Chen's theorem (Theorem 11).

\subsection{Piecewise linear cubature}

The following theorem assures the existence of a cubature formula on Wiener space composed of continuous piecewise linear paths.

Theorem 18 For each positive integer $m$, there exist $n$ paths $w_{1}, \ldots, w_{n} \in C_{0}^{0}\left(\mathbb{R} \oplus \mathbb{R}^{d}\right)$, which are piecewise linear and $n$ positive weights $\lambda_{1}, \ldots, \lambda_{n}$ whose sum is 1 that satisfy $n \leq|\mathscr{A}(m)|$ and

$$
\mathrm{E}\left[\pi_{m}(S(B))\right]=\sum_{i=1}^{n} \lambda_{i} \pi_{m}\left(S\left(w_{i}\right)\right) .
$$

The statement still holds even if we require $w_{i}^{0}(t)=t$ for $i=1, \ldots, n$ and $0 \leq t \leq 1$.

Proof From Theorem 17 and Remark 3 (also from Proposition 14 if we want $\left.w^{0}(t)=t\right)$, we can easily deduce that there exist a set of at most $2|\mathscr{A}(m)|$ continuous piecewise linear paths whose truncated (by $\pi_{m}$ ) signatures convex hull contains $\mathrm{E}\left[\pi_{m}(S(B))\right]$ (in its relative interior). Rigorously, we can apply the same argument as the proof of Theorem 4. Finally, by Carathéodory's theorem, $n \leq|\mathscr{A}(m)|$ can actually be achieved.

Based on this theorem, it is sufficient for us to look for cubature formula within piecewise linear paths. Our approach to construction of a piecewise linear cubature is an application of "Monte Carlo cubature construction" [10]. Of course, we are not able to generate a Brownian motion and use it as a candidate for sample points of cubature formulas, because it is not a BV path and it cannot 
be implemented on computers anyway. However, the methods in [10] are still applicable here as we see in the following proposition.

Proposition 19 Let $m$ be a positive integer. Then, for a sufficiently large $M$ (the lower bound of $M$ depends on $m$ ), the following statement holds:

Let a sequence of continuous piecewise linear paths $w_{1}, w_{2}, \ldots$ be generated identically and independently. Assume also each $w_{i}$ satisfies that

- $w_{i}$ is a path that linearly connects points $w_{i}(k / M)(0 \leq k \leq M)$;

- one of (a) and (b) holds:

(a) $w_{i}^{0}(t)=t(0 \leq t \leq 1)$ holds;

(b) $w_{i}^{0}(0)=0$ and $w_{i}^{0}(1)=1$ hold, and $w_{i}^{0}\left(\frac{k}{M}\right)-w_{i}^{0}\left(\frac{k-1}{M}\right)(1 \leq k \leq M-1)$ are independent random variables and have a density on $\mathbb{R}$ which is positive almost everywhere;

- random variables $w_{i}^{j}\left(\frac{k}{M}\right)-w_{i}^{j}\left(\frac{k-1}{M}\right)(1 \leq j \leq d, 1 \leq k \leq M)$ are independent (also from the ones of zero-th coordinate) and have a density on $\mathbb{R}$, which is positive almost everywhere.

Then, with probability one, there exists an $N$ such that a subset of $\left\{w_{1}, w_{2}, \ldots, w_{N}\right\}$ can construct a cubature on Wiener space of degree $m$.

Proof Take $M$ as large as we can find (at most) $2|\mathscr{A}(m)|$ piecewise linear paths (denoted by $\left.\tilde{w}_{\ell}\right)$ with at most $M$ linear segments such that conv $\left\{\pi_{m}\left(S\left(\tilde{w}_{\ell}\right)\right)\right\}_{\ell}$ contains $\mathrm{E}\left[\pi_{m}(S(B))\right]$ in its relative interior. The existence of an $M$ is assured by the proof of Theorem 18 (or see Remark 3, Theorem 17, and Remark 4).

From (11), the truncated signature of each $w_{i}$ is a polynomial of random variables $w_{i}^{j}\left(\frac{k}{M}\right)-w_{i}^{j}\left(\frac{k-1}{M}\right) \quad(0 \leq j \leq d, 1 \leq k \leq M,(j, k) \neq(0, M))$. Our assumption assures that these variables take values in every neighborhood of some point with a positive probability. In particular, it implies that

$$
\mathrm{P}\left(\left\|\pi_{m}\left(S\left(\tilde{w}_{\ell}\right)\right)-\pi_{m}\left(S\left(w_{i}\right)\right)\right\|<\varepsilon\right)>0,
$$

where $\|\cdot\|$ is the Euclidean norm on $T^{(m)}(E) \simeq F$, holds for each $i, \ell$ and $\varepsilon>0$. Note that the left-hand side probability does not depend on $i$ by i.i.d. assumption. Therefore, the argument in the proof of Theorem 4 holds here again and we obtain the desired assertion.

Remark 5 For the scheme (b), the condition $w_{i}^{0}(1)=1$ is necessary to assure that each $\pi_{m}\left(S\left(w_{i}\right)\right)$ is contained in $G$ defined in Sect. 4.3.

Note that the generation rule of sample paths in this proposition is just one of infinitely many possible examples. We may alternatively, for instance, directly generate $w_{i}^{j}(k / M)$ independently. 
Proposition 19 assures only the existence of "some large" $M$ and $N$, so it might be numerically hard to find cubature formulas from this approach. However, it is beneficial to know that the construction can be reduced at least to the stage of machine power.

\subsection{Numerical experiments}

Explaining our simple numerical method based on a Monte Carlo approach we first describe the algorithm for computing the signature of piecewise linear paths, and then present our Monte Carlo approach and its result in some pairs of $(d, m)$.

Calculation of signature Note that hereafter $d$ and $m$ are regarded as already given parameters. For positive integers $M$ and $N$, we generate $N$ piecewise linear paths (denoted by $w_{1}, \ldots, w_{N}$ ) with $M$ intervals of time (see Proposition 19). The time complexity of this paths generation is $\mathrm{O}(N M d)$. Then, we address each component of generated paths by

$$
\operatorname{PATH}[i, j, k]:=w_{i}^{k}\left(\frac{j}{M}\right)-w_{i}^{k}\left(\frac{j-1}{M}\right) \quad(1 \leq i \leq N, 1 \leq j \leq M, 0 \leq k \leq d) .
$$

In all the experiments, we generated $\operatorname{PATH}[i, j, k]$ (with $k \neq 0$ ) so that it follows the centered normal distribution of variance $1 / M$. We set PATH $[i, j, 0]=1 / M$ for each $i, j$ and denote $w_{i}$ by just writing PATH[i] for each $i$.

As we consider not so large $M$ in this study, we calculate the signature of generated paths by a simple dynamic programming (Algorithm 1). In the algorithm, we calculate the signature of $w_{i}$ over $[0, k / M]$ for $k=1, \ldots, M$, by using the expression (11). The time complexity of this algorithm is $\mathrm{O}\left(M|\mathscr{A}(m)|^{2}\right)$, though a pruning of possible multiindices $(\alpha, \beta)$ helps a bit. 


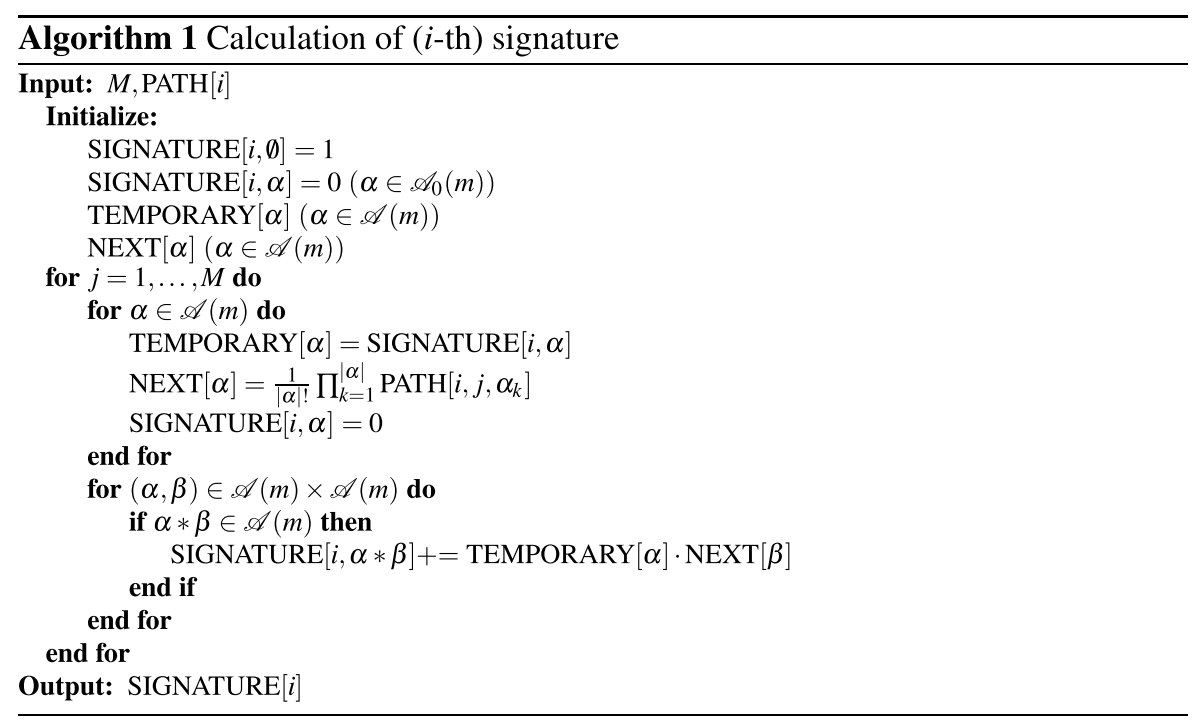

Monte Carlo approach In the approach based on Monte Carlo sampling, we simply generate many paths and determine by solving an LP problem whether or not we can construct a cubature formula of desired degree from generated paths.

The part of solving an LP problem was performed using IBM ILOG CPLEX Optimization Studio (https://www.ibm.com/analytics/cplex-optimizer, version 12.10; CPLEX hereafter). From Proposition 19, for a sufficiently large $N$ we can construct a cubature formula using a subset of paths $\left\{w_{1}, \ldots, w_{N}\right\}$.

We conducted experiments for six cases $(d, m)=(2,3),(3,3),(4,3),(2,5),(3,5),(2,7)$ and for each $(d, m)$, set $N=2|\mathscr{A}(m)|, 4|\mathscr{A}(m)|, 8|\mathscr{A}(m)|, M=2,4,8,16,32$ and examined if we could construct a cubature formula by using CPLEX (note that $|\mathscr{A}(m)|$ depends on $d)$. The following tables show how many times out of 10 trials we successfully obtained cubature formula. Blanks in the tables mean that the corresponding experiments were not performed because we already got 10 successes out of 10 with a smaller $N$.

From these results, one may expect that the change in $m$ is more essential than $d$ where we need larger number of partitions and ratio $N /|\mathscr{A}(m)|$ as $m$ gets larger, though more experiments are necessary.

\section{Concluding remarks}

In this paper, we have demonstrated that piecewise linear cubature formula can be constructed on Wiener space through a Monte Carlo sampling and an LP problem. Our construction is supported by the technical contribution, which extends stochastic Tchakaloff's theorem using our characterization of the distribution of 
Stratonovich iterated integrals. We confirmed that for small pairs of $(d, m)$ our algorithm actually works in numerical experiments.

Although we have shown that one can theoretically construct cubature formulas of any dimension and degree, the number of paths used in our construction only attains the Tchakaloff bound, and therefore it requires too much computational cost for large $(d, m)$ in practice. Therefore, we may consider reducing the number of paths by using additional optimization techniques.

\section{A Proof of Lemma 16}

Here, we give an elementary proof of Lemma 16.

Proof This proof exploits several arguments in [12, Chapter 5]. First, we prove the result for the Ito integrals. Define

$$
\mathscr{A}:=\{\alpha \in \mathscr{A} \mid \alpha \text { contains at least one nonzero index }\} .
$$

We define for $t \geq 0$ that $\mathscr{I}_{t}^{\infty}:=1$ and

$$
\mathscr{I}_{t}^{\alpha}:=\int_{0<t_{1}<\cdots<t_{k}<t} \mathrm{~d} B_{t_{1}}^{i_{1}} \cdots \mathrm{d} B_{t_{k}}^{i_{k}}
$$

for each $\alpha=\left(i_{1}, \ldots, i_{k}\right) \in \mathscr{A}$. We also define $\alpha-:=\left(i_{1}, \ldots, i_{k-1}\right)$ and $s(\alpha)=i_{k}$ for $\alpha \neq \emptyset$. Note that it holds that

$$
\mathscr{I}_{t}^{\alpha}=\int_{0}^{t} \mathscr{I}_{r}^{\alpha-} \mathrm{d} B_{r}^{s(\alpha)} .
$$

For a multiindex $\alpha=\left(i_{1}, \ldots, i_{k}\right) \in \mathscr{A}$, let $\alpha^{+}$be the sequence defined by nonzero indices of $\alpha$. For example, if $\alpha=(0,2,0,1,1,0)$, then $\alpha^{+}=(2,1,1)$.

Let $u=\left(u_{t}\right)_{t \geq 0}$ be a progressively measurable and second mean integrable stochastic process. Then, for each index $i \neq 0$,

$$
\int_{0}^{t}\left(\int_{0}^{s} u_{r} \mathrm{~d} B_{r}^{i}\right) \mathrm{d} s=\int_{0}^{t}\left(\int_{0}^{t} u_{r} 1_{\{s>r\}}(r, s) \mathrm{d} s\right) \mathrm{d} B_{r}^{i}=\int_{0}^{t}(t-r) u_{r} \mathrm{~d} B_{r}^{i}
$$

holds. By using Ito isometry and this relation repeatedly, we can show that

$$
\mathrm{E}\left[\mathscr{I}_{t}^{\alpha} \mathscr{I}_{t}^{\beta}\right]=0, \quad \text { if } \alpha, \beta \in \mathscr{A} \text { and } s\left(\alpha^{+}\right) \neq s\left(\beta^{+}\right) .
$$

Indeed, in the case $k$-times zeros appear in the suffix of $\alpha$, we can show inductively 


$$
\begin{aligned}
& \int_{0<t_{1}<\cdots<t_{k}<t}\left(\int_{0}^{t_{1}} u_{r} \mathrm{~d} B_{r}^{i}\right) \mathrm{d} t_{1} \cdots \mathrm{d} t_{k} \\
& =\cdots=\int_{0<t_{j}<\cdots<t_{k}<t}\left(\int_{0}^{t_{j}} \frac{\left(t_{j}-r\right)^{j-1}}{(j-1) !} u_{r} \mathrm{~d} B_{r}^{i}\right) \mathrm{d} t_{j} \cdots \mathrm{d} t_{k} \\
& =\cdots=\int_{0}^{t} \frac{(t-r)^{k}}{k !} u_{r} \mathrm{~d} B_{r}^{i},
\end{aligned}
$$

so, we can finally apply the following Ito isometry:

for progressively measurable and second mean integrable stochastic process $u$ and $v$, it holds that

$$
\mathrm{E}\left[\left(\int_{0}^{t} u_{s} \mathrm{~d} B_{s}^{i}\right)\left(\int_{0}^{t} v_{s} \mathrm{~d} B_{s}^{j}\right)\right]=\delta^{i j} \mathrm{E}\left[\int_{0}^{t} u_{s} v_{s} \mathrm{~d} s\right],
$$

where $i, j \in\{1, \ldots, d\}$ and $\delta^{i j}$ is Kronecker's delta.

We then prove a stronger assertion than (12), which states

$$
\mathrm{E}\left[\mathscr{I}_{t}^{\alpha} \mathscr{I}_{t}^{\beta}\right]=0, \quad \text { if } \alpha \text { or } \beta \in \mathscr{A}, \text { and } \alpha^{+} \neq \beta^{+} \text {. }
$$

Let $\alpha=\tilde{\alpha} *(i, \underbrace{0, \ldots, 0})$ and $\beta=\tilde{\beta} *(j, \underbrace{0, \ldots, 0})$. It suffices to consider the case $i=j \neq 0$, and we have

$$
\underbrace{0, \ldots, 0}_{k \text { times }} \text { and } \beta=\tilde{\beta} *(j, \underbrace{0, \ldots, 0}_{\ell \text { times }}
$$

$$
\begin{aligned}
& \mathrm{E}\left[\mathscr{I}_{t}^{\alpha} \mathscr{I}_{t}^{\beta}\right]=\mathrm{E}\left[\left(\int_{0}^{t} \frac{(t-s)^{k}}{k !} \mathscr{I}_{s}^{\tilde{\alpha}} \mathrm{d} B_{s}^{i}\right)\left(\int_{0}^{t} \frac{(t-s)^{\ell}}{\ell !} \mathscr{I}_{s} \mathrm{~d} B_{s}^{i}\right)\right] \\
& =\mathrm{E}\left[\int_{0}^{t} \frac{(t-s)^{k+\ell}}{k ! \ell !} \mathscr{I}_{s}^{\tilde{\alpha}} \mathscr{I}_{s}^{\tilde{\beta}} \mathrm{d} s\right] \\
& =\int_{0}^{t} \frac{(t-s)^{k+\ell}}{k ! \ell !} \mathrm{E}\left[\begin{array}{c}
\tilde{I_{s}} \mathscr{I}_{s}^{\tilde{\beta}} \\
{ }^{2}
\end{array}\right] \mathrm{d} s \text {. }
\end{aligned}
$$

Therefore, by an inductive argument, it only remains to prove that $\mathrm{E}\left[\mathscr{I}_{t}^{\alpha} \mathscr{I}_{t}^{\beta}\right]=0$ in the case $\alpha$ or $\beta$ only contain zeros and $\alpha^{+} \neq \beta^{+}$, but this case is trivial as the one is a constant and the other's expectation becomes zero from (13). We now have completed the proof of (14).

From these results, we have the decomposition

$$
\mathrm{E}\left[\left(\sum_{\alpha \in \mathscr{A}} c_{\alpha} \mathscr{I}_{t}^{\alpha}\right)^{2}\right]=\sum_{\beta \in \mathscr{A}^{+}} \mathrm{E}\left[\left(\sum_{\alpha \in \mathscr{A}, \alpha^{+}=\beta} c_{\alpha} \mathscr{I}_{t}^{\alpha}\right)^{2}\right],
$$

where all but finite $c_{\alpha}$ equal to zero and $\mathscr{A}^{+}$denotes the set of all multiindices that contain no zeros. Therefore, it suffices to consider sums of the form $\sum_{i=1}^{n} c_{i} \mathscr{I}_{t}^{\alpha_{i}}$, with $c_{1}, \ldots, c_{n} \in \mathbb{R}$ and $\alpha_{1}^{+}=\cdots=\alpha_{n}^{+} \neq \emptyset$. Note that $\alpha_{1}, \ldots, \alpha_{n}$ can be taken pairwaise 
different. One may assume this sum almost surely equals to zero. Next, we prove that $\sum_{i=1}^{n} c_{i} I^{\alpha_{i}}(w)_{0, t}$ is also zero for the $w$ with $w(s)=s\left(I^{\alpha}(w)_{s, t}\right.$ is $S(w)_{s, t}$ entry corresponding to the multiindex $\alpha)$. Let each $\alpha_{i}$ have the form $\alpha_{i}=\beta_{i} *(j, 0, \ldots, 0)$,

where $j \neq 0$ is an index independent of $i$.

$k_{i}$ times

From (13), we have

$$
\begin{aligned}
\mathrm{E}\left[\left(\sum_{i=1}^{n} c_{i} \mathscr{I}_{t}^{\alpha_{i}}\right)^{2}\right] & =\mathrm{E}\left[\left(\int_{0}^{t} \sum_{i=1}^{n} \frac{(t-r)^{k_{i}}}{k_{i} !} c_{i} \mathscr{I}_{r}^{\beta_{i}} \mathrm{~d} B_{r}^{j}\right)^{2}\right] \\
& =\mathrm{E}\left[\int_{0}^{t}\left(\sum_{i=1}^{n} \frac{(t-r)^{k_{i}}}{k_{i} !} c_{i} \mathscr{I}_{r}^{\beta_{i}}\right)^{2} \mathrm{~d} r\right] \\
& =\int_{0}^{t} \mathrm{E}\left[\left(\sum_{i=1}^{n} \frac{(t-r)^{k_{i}}}{k_{i} !} c_{i} \mathscr{I}_{r}^{\beta_{i}}\right)^{2}\right] \mathrm{d} r .
\end{aligned}
$$

If the left-hand side is zero, the integrand in the right-hand side is also zero for all $0 \leq r \leq t$ because it is continuous in $r$.

Here, we note that the deterministic counterpart of (13) holds. Indeed, if $u=\left(u_{t}\right)_{t \geq 0}$ is a continuous $\mathbb{R}$-valued path, then for a $w \in C_{0}^{0}\left([0,1] ; \mathbb{R} \oplus \mathbb{R}^{d}\right)$ with $w^{0}(t)=t$, we have

$$
\begin{aligned}
& \int_{0<t_{1}<\cdots<t_{k}<t}\left(\int_{0}^{t_{1}} u_{r} \mathrm{~d} w_{r}^{i}\right) \mathrm{d} t_{1} \cdots \mathrm{d} t_{k} \\
& =\cdots=\int_{0<t_{j}<\cdots<t_{k}<t}\left(\int_{0}^{t_{j}} \frac{\left(t_{j}-r\right)^{j-1}}{(j-1) !} u_{r} \mathrm{~d} w_{r}^{i}\right) \mathrm{d} t_{j} \cdots \mathrm{d} t_{k} \\
& =\cdots=\int_{0}^{t} \frac{(t-r)^{k}}{k !} u_{r} \mathrm{~d} w_{r}^{i} .
\end{aligned}
$$

Therefore, we obtain

$$
\sum_{i=1}^{n} c_{i} I^{\alpha_{i}}(w)_{0, t}=\int_{0}^{t}\left(\frac{(t-r)^{k_{i}}}{k_{i} !} c_{i} I^{\beta_{i}}(w)_{0, r}\right) \mathrm{d} w_{r}^{j} .
$$

As we have already obtained $\sum_{i=1}^{n} \frac{(t-r)^{k_{i}}}{k_{i} !} c_{i} \mathscr{S}_{r}^{\beta_{i}}=0$ for $0 \leq r \leq t$, the problem reduces to that of $\beta_{1}, \ldots, \beta_{n}$. Therefore, by inductive arguments with respect to $\left|\alpha_{1}^{+}\right|$we can prove that

$$
\sum_{i=1}^{n} c_{i} \mathscr{I}_{t}^{\alpha_{i}}=0 \Longrightarrow \sum_{i=1}^{n} c_{i} I^{\alpha_{i}}(w)_{0, t}=0,
$$

where the base step is trivial as it only contains the integral with respect to time. 
Though we have to extend this result when $t=1$ to all the $w$ satisfying only $w^{0}(1)=1$, it can be proved by using perturbation arguments. More precisely, we can prove the result for piecewise linear paths with $w^{0}(1)=1$ as $\sum_{i} c_{i} I^{\alpha_{i}}(w)$ is a polynomial of increments in the path that equals to zero at infinitely many points (if $w^{0}$ is monotone increasing, then we can reparametrize it so that $\left.w^{0}(t)=t\right)$. Indeed, proving for piecewise linear paths is sufficient [7, Theorem 7.28].

It remains to modify the result for Stratonovich integrals. The relation between multiple Stratonovich integrals and Ito integrals is known as follows [12]:

$$
\begin{aligned}
I^{\emptyset}(B)_{0, t} & =\mathscr{I}_{t}^{\emptyset}=1, \\
I^{(i)}(B)_{0, t} & =\mathscr{I}_{t}^{(i)} \quad(i=0,1, \ldots, d), \\
I^{\alpha}(B)_{0, t} & =\int_{0}^{t} I^{\alpha-}(B)_{0, r} \mathrm{~d} B_{r}^{s(\alpha)}+\frac{1}{2} \cdot 1_{\{s(\alpha)=s(\alpha-) \neq 0\}} \int_{0}^{t} I^{(\alpha-)-}(B)_{0, r} \mathrm{~d} r \quad(|\alpha| \geq 2) .
\end{aligned}
$$

Therefore, each $I^{\alpha}(B)_{0, t}$ is represented as a positive combination of $\mathscr{I}_{t}^{\beta}$ such that $\beta$ can be acquired by replacing two consecutive same nonzero indices by a zero some times.

The assumption $\sum_{i=1}^{n} c_{i} I^{\alpha_{i}}(B)=0$ holds almost surely $\left(c_{i} \neq 0\right.$ for all $\left.i\right)$. We shall again prove $\sum_{i=1}^{n} c_{i} I^{\alpha_{i}}(w)$ by inductive arguments.

Let $i_{0} \in \arg \max _{i}\left|\alpha_{i}^{+}\right|$and $\beta:=\alpha_{i_{0}}^{+}$. Then, by the above expansion, $\sum_{i=1}^{n} c_{i} I^{\alpha_{i}}(B)$ can be rewritten as a sum of Ito integrals. In particular,

$$
\sum_{i=1}^{n} c_{i} I^{\alpha_{i}}(B)-\sum_{i: \alpha_{i}^{+}=\beta} c_{i} \mathscr{I}_{1}^{\alpha_{i}}
$$

is represented as a weighted sum of $\mathscr{I}_{1}^{\alpha}$ with $\alpha^{+} \neq \beta$. Therefore, $\sum_{i: \alpha_{i}^{+}=\beta} c_{i} \mathscr{I}_{1}^{\alpha_{i}}=0$ holds almost surely, and so $\sum_{i: \alpha_{i}^{+}=\beta} c_{i} I^{\alpha_{i}}(w)=0$ holds for all the valid $w$.

From Theorem 11 and Proposition 12, with probability one there exists some (random) $w$ such that $\pi_{n}(S(B))=\pi_{n}(S(w))$, where sufficiently large $n$ is taken. By using such $w$, we obtain

$$
\sum_{i: \alpha_{i}^{+}=\beta} c_{i} I^{\alpha_{i}}(B)=\sum_{i: \alpha_{i}^{+}=\beta} c_{i} I^{\alpha_{i}}(w)=0 .
$$

Therefore, if $\sum_{i=1}^{n} c_{i} I^{\alpha_{i}}(B)=0$ holds almost surely, then we can prove inductively (with respect to some order over $\mathscr{A}^{+}$), for each $\beta \in \mathscr{A}^{+}$and $w$,

$$
\sum_{i: \alpha_{i}^{+}=\beta} c_{i} I^{\alpha_{i}}(w)=0, \quad \sum_{i: \alpha_{i}^{+}=\beta} c_{i} I^{\alpha_{i}}(B)=0 .
$$

By considering the sum, we obtain $\sum_{i=1}^{n} c_{i} I^{\alpha_{i}}(w)=0$ and the proof is completed.

Remark 6 In the last part of the above proof, we have essentially used the assertion (actually the inverse also follows from the above proof) 


$$
\sum_{\alpha} c_{\alpha} \mathscr{I}_{t}^{\alpha}=0 \quad \Longrightarrow \quad \sum_{\alpha} c_{\alpha} I(B)_{0, t}=0
$$

Although we have proved it via Lie-algebraic arguments, which exploit Theorem 11 and Proposition 12 , it can be directly proved by repeatedly using the relations (13) and (16).

Acknowledgements The first author would like to thank Terry Lyons for his insightful comments on Proposition 13. The authors would like to thank Enago (www.enago.jp) for the manuscript review and editing support. The authors are also grateful to the reviewers for their careful reading and constructive feedback. This study was supported by the Japan Society for the Promotion of Science with KAKENHI (17K14241 to K.T.).

Open Access This article is licensed under a Creative Commons Attribution 4.0 International License, which permits use, sharing, adaptation, distribution and reproduction in any medium or format, as long as you give appropriate credit to the original author(s) and the source, provide a link to the Creative Commons licence, and indicate if changes were made. The images or other third party material in this article are included in the article's Creative Commons licence, unless indicated otherwise in a credit line to the material. If material is not included in the article's Creative Commons licence and your intended use is not permitted by statutory regulation or exceeds the permitted use, you will need to obtain permission directly from the copyright holder. To view a copy of this licence, visit http://creativecommons.org/licen ses/by/4.0/.

\section{References}

1. Baudoin, F.: An introduction to the geometry of stochastic flows. World Scientific, Singapore (2004)

2. Bayer, C., Teichmann, J.: The proof of Tchakaloff's theorem. Proc. Am. Math. Soc. 134(10), 30353040 (2006)

3. Bonnice, W., Klee, V.L.: The generation of convex hulls. Math. Ann. 152(1), 1-29 (1963)

4. Chen, K.T.: Integration of paths, geometric invariants and a generalized Baker-Hausdorff formula. Ann. Math. 65, 163-178 (1957)

5. Crisan, D., Ghazali, S.: On the convergence rates of a general class of weak approximations of SDEs. In: Stochastic Differential Equations: Theory And Applications: A Volume in Honor of Professor Boris L Rozovskii, pp. 221-248. World Scientific, Singapore (2007)

6. Friz, P.K., Hairer, M.: A course on rough paths: with an introduction to regularity structures. Springer, Berlin (2014)

7. Friz, P.K., Victoir, N.B.: Multidimensional stochastic processes as rough paths: theory and applications, vol. 120. Cambridge University Press, Cambridge (2010)

8. Gyurkó, L.G., Lyons, T.: Efficient and practical implementations of cubature on wiener space. In: Stochastic analysis 2010, pp. 73-111. Springer, Berlin (2011)

9. Hairer, M.: On Malliavin's proof of Hörmander's theorem. Bull. Sci. Math. 135(6-7), 650-666 (2011)

10. Hayakawa, S.: Monte Carlo cubature construction. Jpn. J. Indus. Appl. Math. (2020). https://doi.org/ 10.1007/s13160-020-00451-X

11. Ikeda, N., Watanabe, S.: Stochastic differential equations and diffusion processes, vol. 24. NorthHolland Mathematical Library, Amsterdam (1989)

12. Kloeden, P.E., Platen, E.: Numerical solution of stochastic differential equations. Springer, Berlin (1992)

13. Kusuoka, S.: Approximation of expectation of diffusion process and mathematical finance. In: Taniguchi Conference on Mathematics Nara '98, pp. 147-165. Mathematical Society of Japan (2001)

14. Kusuoka, S.: Malliavin calculus revisited. J. Math. Sci. 10(2), 261-277 (2003) 
15. Kusuoka, S.: Approximation of expectation of diffusion processes based on Lie algebra and Malliavin calculus. Adv. Math. Econ. 6, 69-83 (2004)

16. Kusuoka, S., Stroock, D.: Applications of Malliavin calculus. III. J. Fac. Sci. 1A(34), 391-442 (1987)

17. Litterer, C., Lyons, T.: High order recombination and an application to cubature on Wiener space. Ann. Appl. Probab. 22(4), 1301-1327 (2012)

18. Lyons, T.: Differential equations driven by rough signals. Rev. Mat. Iberoam. 14(2), 215-310 (1998)

19. Lyons, T., Victoir, N.: Cubature on Wiener space. Proc. R. Soc. Lond. Ser. A 460, 169-198 (2004)

20. Ninomiya, M., Ninomiya, S.: A new higher-order weak approximation scheme for stochastic differential equations and the Runge-Kutta method. Finance Stoch 13(3), 415-443 (2009)

21. Ninomiya, S., Shinozaki, Y.: Higher-order discretization methods of forward-backward SDEs using KLNV-scheme and their applications to XVA pricing. Appl. Math. Finance 26(3), 257-292 (2019)

22. Ninomiya, S., Victoir, N.: Weak approximation of stochastic differential equations and application to derivative pricing. Appl. Math. Finance 15(2), 107-121 (2008)

23. Ninorniya, S.: A partial sampling method applied to the Kusuoka approximation. Monte Carlo Methods Appl. 9(1), 27-38 (2003)

24. Oshima, K., Teichmann, J., Velušček, D.: A new extrapolation method for weak approximation schemes with applications. Ann. Appl. Probab. 22(3), 1008-1045 (2012)

25. Piazzon, F., Sommariva, A., Vianello, M.: Caratheodory-Tchakaloff subsampling. Dolomites Res. Notes Approx. 10, 5-15 (2017)

26. Reutenauer, C.: Free Lie algebras. Oxford Science, Oxford (1993)

27. Rousseeuw, P.J., Ruts, I.: The depth function of a population distribution. Metrika 49(3), 213-244 (1999)

28. Sawa, M., Hirao, M., Kageyama, S.: Euclidean Design Theory. Springer, Berlin (2019)

29. Shinozaki, Y.: Construction of a third-order K-scheme and its application to financial models. SIAM J. Financ. Math. 8(1), 901-932 (2017)

30. Steinitz, E.: Bedingt konvergente reihen und konvexe systeme. (schluß.). J. die reine Angew. Math. 146, 1-52 (1916)

31. Tchakaloff, V.: Formules de cubature mécanique à coefficients non négatifs. Bull. Sci. Math. 81, 123-134 (1957)

32. Tchernychova, M.: Carathéodory cubature measures. Ph.D. thesis, University of Oxford (2015)

Publisher's Note Springer Nature remains neutral with regard to jurisdictional claims in published maps and institutional affiliations. 